\title{
MOVIMIENTO SUPERFICIAL DEL GLACIAR ROCOSO DE LAS ARGUALAS
}

\author{
Serrano, E.(1); Sanjosé, J.J.(2); Sillo, F.(1); Agudo, C.(3)
}

(1) Dpto. de Geografía, Urbanismo y O. T. Universidad de Cantabria.

(2) Dpto. de Ingeniería Geográfica y Expresión Gráfica. Universidad de Cantabria.

(3) Dpto. de Geografía. Universidad Autónoma de Madrid.

\begin{abstract}
RESUMEN.- El glaciar rocoso de las Argualas es activo en la actualidad

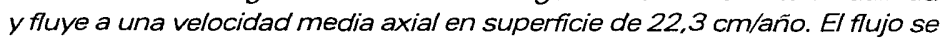
ha determinado mediantelas técnicas de auscultación topográfica realizadas entre los años 1991 y 1994. Esta técnica ha permitido complementar el ànálisis geomorfológico, profundizado en el estudio dela dinámica superficial del glaciar.
\end{abstract}

ABSTRACT.-At present the Argualas rock glacier is active and it flows to a surface axial mean velocity of $22,3 \mathrm{~cm} /$ year. The flow velocities have been determinated by surveying techniques made between 1991 and 1994. This technic complements the geomorphologicalobservations and deepens in the knowledge of the glacier surface dynamics.

Key-words: Rock glaciers, glacial and periglacial procceses; surveying control, Pyrenees.

\section{Introducción}

El glaciar rocoso de las Argualas, situado en el macizo del mismo nombre, en la alta montaña del valle de Tena (Pirineo aragonés), ha sido objeto de estudios de detalle desde el año 1988. Estos se han centrado en su geomorfología y en el análisis superficial de clastos, con el objetivo de caracterizarlo y conocer su dinámismo y cronología (Martínez de Pisón y Arenillas, 1989; Serrano y Rubio, 1989). Serrano y Rubio (1989) apuntan la hipótesis de un glaciar rocoso activo con caracteres de forma parcialmente heredada, por lo que se hizo necesario un estudio de detalle que permitiera confirmar con precisión su dinámica actual. Para ello se han iniciado trabajos fotogramétricos, de auscultación topográfica y sondeos geoeléctricos. Todos los trabajos tienen por objetivo establecer de forma precisa sus características geomorfológicas, conociendo el tipo de glaciar rocoso, su evolución y dinámica actual. 
PIRINEOS 145-146

Tabla 1. Glaciar rocoso de las Argualas.

\begin{tabular}{|c|c|}
\hline \multirow[t]{4}{*}{ LOCALIZACION } & Pirineo de Huesca \\
\hline & Cuenca: Gállego \\
\hline & Rio: Pondiellos \\
\hline & $\begin{array}{l}\text { Macizo: Argualas } \\
(3.071 \mathrm{~m} .)\end{array}$ \\
\hline TIPO DE GLACIAR & $\begin{array}{l}\text { Glaciar rocoso } \\
\text { activo }\end{array}$ \\
\hline SUPERFICIE & 35 Has. \\
\hline LONGITUD & $750 \mathrm{~m}$ \\
\hline ANCHURA & $400 \mathrm{~m}$ \\
\hline \multirow[t]{3}{*}{ ALTITUDES } & Máxima: $2.700 \mathrm{~m}$. \\
\hline & Minima: $2.590 \mathrm{~m}$ \\
\hline & Cumbres:3.032 m. \\
\hline ORIENTACION & Noroeste \\
\hline PENDIENTE MEDIA & $25^{\circ}$ \\
\hline VELOCIDAD media axial & $22,3 \mathrm{~cm} /$ año \\
\hline TIPO DE ALIMENTACION & $\begin{array}{l}\text { Aludes de piedra y } \\
\text { nieve }\end{array}$ \\
\hline
\end{tabular}

En el verano de 1991 se instaló la malla de puntos para el control topográfico, realizándose controles en sucesivas campañas los años 1993 y 1994. En la actualidad se cuenta con un mapa topográfico escala 1/5.000 del circo donde se sitúa el glaciar, así como de sondeos geoeléctricos cuyos resultados están en fase de elaboración.

El glaciar rocoso se ubica en el circo Noroeste de Argualas, entre las cumbres de Algas (3.03' m.) y Garmo Negro (3.072 m.), a 2.590-2.730 $\mathrm{m}$. de altitud. Constituye una masa caótica de clastos heterométricos en forma de lengua curvada al N.W. que engloba surcos longitudinales $y$ arcos y surcos transversales. El frente está constituido por un talud de 20$25 \mathrm{~m}$. y $42^{\circ}$ de pendiente en el que afloran los materiales menos groseros. Se pueden diferenciar tres sectores:

- de alimentación, con acumulación de clastos por gravedad y aludes de nieve y roca que fosilizan los heleros de cabecera y son redistribuidos en un primer momento por procesos periglaciares plenamente activos. 
MOVIMIENTO SUPERFICIAL DEL GLACIAR ROCOSO DE LAS ARGUALAS

- medio, glaciar cubierto en el que dominan los flujos longitudinales y se ha observado la presencia de un núcleo de hięlo sobre el que reposa la masa de clastos.

- inferior, glaciar rocoso s.s., donde se acumulan los materiales con dominio de formas de flujo transversal (Serrano y Rubio, 1989).

\section{Metodología}

Establecidas las características morfológicas mediante la cartografía y el estudio de los materiales superficiales y su disposición, se podía afirmar que existía un dinamismo actual a partir de la observación de la alimentación actual, la frescura e inestabilidad de las formas superficiales, la posición y morfología del frente y la ausencia de líquenes. Para confirmar y cuantificar el movimiento del glaciar rocoso se ha empleado la técnica de auscultación topográfica (San José et al. 1992). El control topográfico es una técnica utilizada habitualmente en el estudio de los glaciares rocosos y ha sido aplicada anteriormente en otros aparatos en Los Alpes, Andes, Rocosas, etc.(Haeberli, 1985; Giardino et al., 1987) con resultados positivos.

En el glaciar rocoso de las Argualas se ha establecido un sistema de señalización sobre la superficie del glaciar, de carácter permanente y solidario, que permite un seguimiento continuado desde tres estaciones externas al mismo, permanentes y estables. Este consta de catorce varillas de 1,80 y $0,80 \mathrm{~cm}$. de longitud con punta de tungsteno y cabezal para soporte del reflector, que forman una malla sobre su superficie. Además, se han colocado dos clavos en un bloque de siete metros de eje mayor localizado sobre la superficie del glaciar, lo que completa los dieciseis puntos de control.

Para el control topográfico se han instalado fuera del glaciar tres referencias $y$ tres estaciones. Las estaciones se ubican en la arista N.W. del Garmo Negro, cuya situación geométrica no es la más apropiada, pero la topografía del entorno del glaciar impide otros estacionamientos teóricamente más idóneos. Con este equipamiento y la observación continuada empleando una estación total Wild TC 2.000, se pretende alcanzar precisiones de 1-2 cms. en la distancia y de 5 a 10 segundos centesimales en los ángulos. Todo ello impide trabajar con precisiones mayores a los $5 \mathrm{~cm}$., por lo que el margen de error lo situamos en los $5 \mathrm{~cm}$./ año. Las medidas anuales que se encuentren en estos márgenes de error son desestimadas en el control total del movimiento, si bien se registran para su control acumulado en una serie mayor de observaciones. 


\section{Resultados}

En la actualidad se cuenta con los datos obtenidos en la campaña de 1993 , en los que se detectó el movimiento del glaciar rocoso entre los años 1991-1993 (tabla 2), y en la de 1994, con resultados de movimientos anuales. En el cuadro adjunto se pueden observar unas velocidades $y$ direcciones de flujo constantes para ambas campañas de control, lo que permite afirmar que los resultados son homogéneos. El análisis de los datos de tres años debe considerarse provisional, y es necesario continuar el control sobre el glaciar rocoso para obtener datos más rigurosos, si bien permite ya observar hechos significativos.

Tabla 2.. Velocidades superficiales del glaciar rocoso de las Argualas.

\begin{tabular}{|c|c|c|c|c|}
\hline \multirow{2}{*}{$\begin{array}{c}\text { VARILLAS } \\
n^{\circ}\end{array}$} & \multicolumn{2}{|c|}{ MOVIMIENTOS 1991-93 } & \multirow{2}{*}{$\begin{array}{l}\text { MOVIMIENTO } \\
\text { 1993-94 } \\
\text { cm/año }\end{array}$} & \multirow{2}{*}{$\begin{array}{c}\text { VELOCIDAD MEDIA } \\
\text { cm/año } \\
1991-1994\end{array}$} \\
\hline & Total & $\mathrm{cm} / \mathrm{año}$ & & \\
\hline 1 & 9 & 4,5 & $5,25^{*}$ & $4,75^{*}$ \\
\hline 2 & 9,5 & 4,75 & $5^{*}$ & $4,8^{*}$ \\
\hline 3 & 27 & 13,5 & 15 & 14 \\
\hline 4 & 44,5 & 22,25 & 24 & 22,8 \\
\hline 5 & 64,5 & 32,25 & 32 & 32,1 \\
\hline 6 & 110 & 55 & 43,5 & 51 \\
\hline 7 & 42,5 & 21,25 & 21,5 & 21,3 \\
\hline 8 & 30 & 15 & 15 & 15 \\
\hline 9 & 12 & 6 & 8 & 6,6 \\
\hline 10 & 46,5 & 23 & 24 & 23,5 \\
\hline 11 & 52 & 26 & 30 & 27,3 \\
\hline 12 & 60 & 30 & 33 & 31 \\
\hline 13 & 58,5 & 29,25 & 35 & 31,16 \\
\hline 14 & 58,5 & 29,25 & 32 & 30,01 \\
\hline 15 & 47 & 23,5 & 26 & 24,33 \\
\hline 16 & 6 & $3^{*}$ & $5^{*}$ & $3,6^{*}$ \\
\hline
\end{tabular}

* La precisión topográfica alcanzada es de $5 \mathrm{~cm}$., por lo que las velocidades inferiores son desestimadas. Se incluyen por su validez cuando se obtengan series más largas de datos.

- Un movimiento superficial diferenciado en sectores (tabla 3). La zona más dinámica es el eje central inferior $(31,5 \mathrm{~cm} / a n ̃ o)$, donde se produce la mayor acumulación de materiales, seguido de el sector axial medio $(29,5 \mathrm{~cm} / a n ̃ o)$ y axial superior $(23,8 \mathrm{~cm} / a n ̃ o)$. Es pues, en el eje del glaciar donde se concentra su dinámica de flujo en la actualidad. 
MOVIMIENTO SUPERFICIAL DEL GLACIAR ROCOSO DE LAS ARGUALAS

- Incremento de la velocidad a medida que nos alejamos de los sectores de alimentación. Una observación por sectores altitudinales permite observar que la velocidad media superficial en el sector más

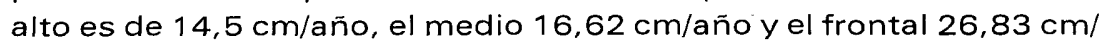
año. Este mismo hecho se observa tanto en el sector axial como en los laterales del glaciar.

Tabla 3. Velocidades medias superficiales por sectores en el glaciar rocoso de las Argualas.

\begin{tabular}{|c|c|c|}
\hline \multicolumn{2}{|r|}{ SECTOR } & $\begin{array}{l}\text { VELOCIDAD MEDIA } \\
\mathrm{cm} / a n ̃ o\end{array}$ \\
\hline & ALTO & 14,5 \\
\hline & MEDIO & 16,62 \\
\hline & FRONTAL & 26,83 \\
\hline A & INFERIOR & 31,5 \\
\hline 1 & MEDIO & 29,5 \\
\hline L & ALTO & 23,8 \\
\hline & APOFISIS & 7,8 \\
\hline $\begin{array}{l}\mathrm{L} \\
\mathrm{A} \\
\mathrm{T}\end{array}$ & ORIENTAL & 14,3 \\
\hline $\begin{array}{l}R \\
A \\
\text { L }\end{array}$ & OCIDENTAL & 13,4 \\
\hline
\end{tabular}

- La velocidad máxima se registra en los sectores frontales, con avances del orden $30 \mathrm{~cm} / a n ̃ o . ~ L a ~ v a r i l l a ~ n^{\circ} 10$ se desestima de este cálculo por estar situada en el frente del glaciar, donde a la inestabilidad del flujo hay que sumar la gravitacional y la derivada de posibles golpes de clastos, aunque este hecho no se ha observado en la campaña del 94.

Los márgenes son los sectores menos activos, si bien conservan el incremento de velocidad con la distancia. La apófisis del margen septentrional registra un movimiento muy limitado, que se incluye en los márgenes de error, pero permite denotar un atenuamiento reciente de su dinámica, dado que las formas se conservan frescas. La diferencia entre las varillas 2 y 3 y la dirección del flujo de la última confirman esta observación. 


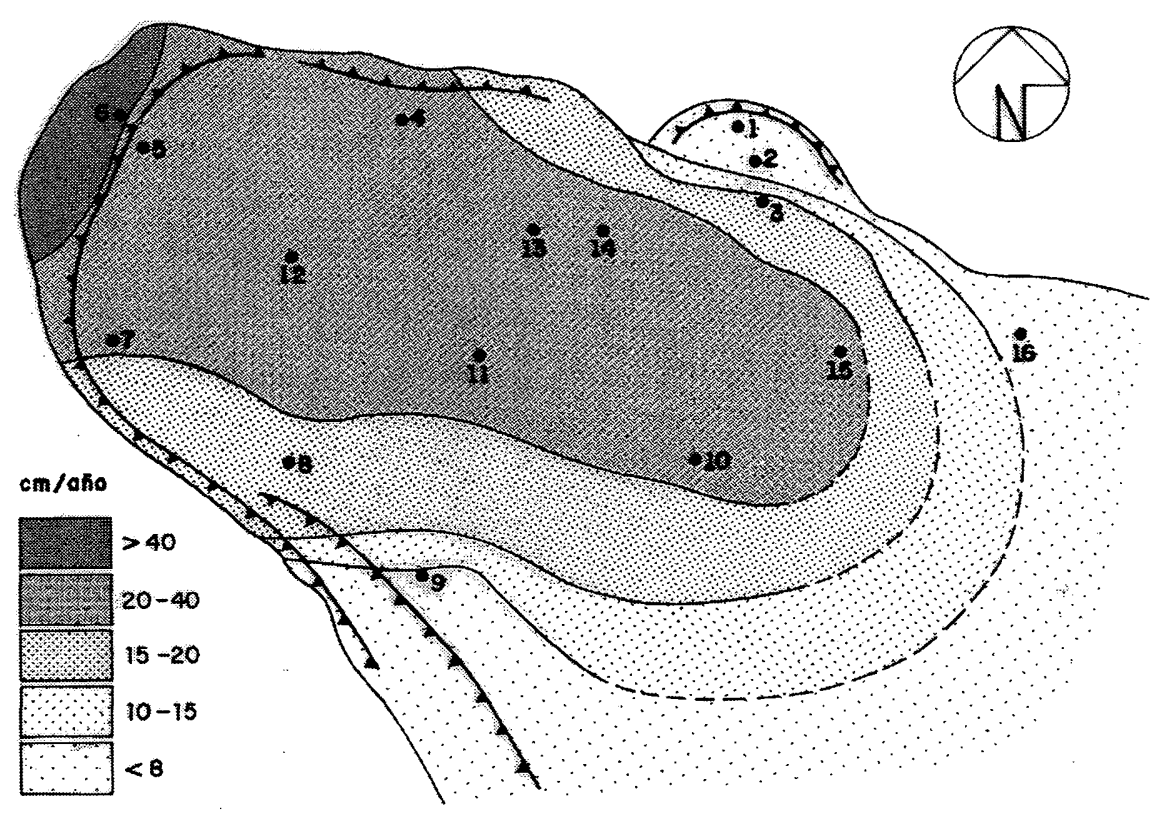

Fig. 1. Mapa de distribución de velocidades de flujo sobre el glaciar rocoso de Las Argualas, con localización de las varillas de control.

- El flujo del glaciar en estos tres años puede considerarse unidireccional hacia el N.W. con apertura en el borde occidental también hacia el oeste. Esto permite deducir una dinámica dirigida por una alimentación de las paredes centrales del circo, procedentes del Pico Garmo Negro y del collado. Esta alimentación es, pues, diferencial entre los sectores centrales, más enérgicos, y los occidentales, menos intensos y dominados por los procesos gravitacionales (conos y taludes de derrubios).

- Las observaciones geomorfológicas y la auscultación realizadas sobre el glaciar rocoso denotan la existencia de plena actividad, si bien es posible observar cambios dinámicos recientes, con sectores en los que su dinámica está atenuada y un eje central muy móvil. El glaciar presenta un avance actual en su frente, sin denotar una ralentización en este sector, donde se acumula la mayor cantidad de clastos, como es característico en los glaciares rocosos estudiados por otros autores. Este hecho concuerda con las orientaciones de los clastos, en los que se refleja una activa orientación preferente en los sectores frontales frente a la apófisis, donde muestra una orientación desigual. 


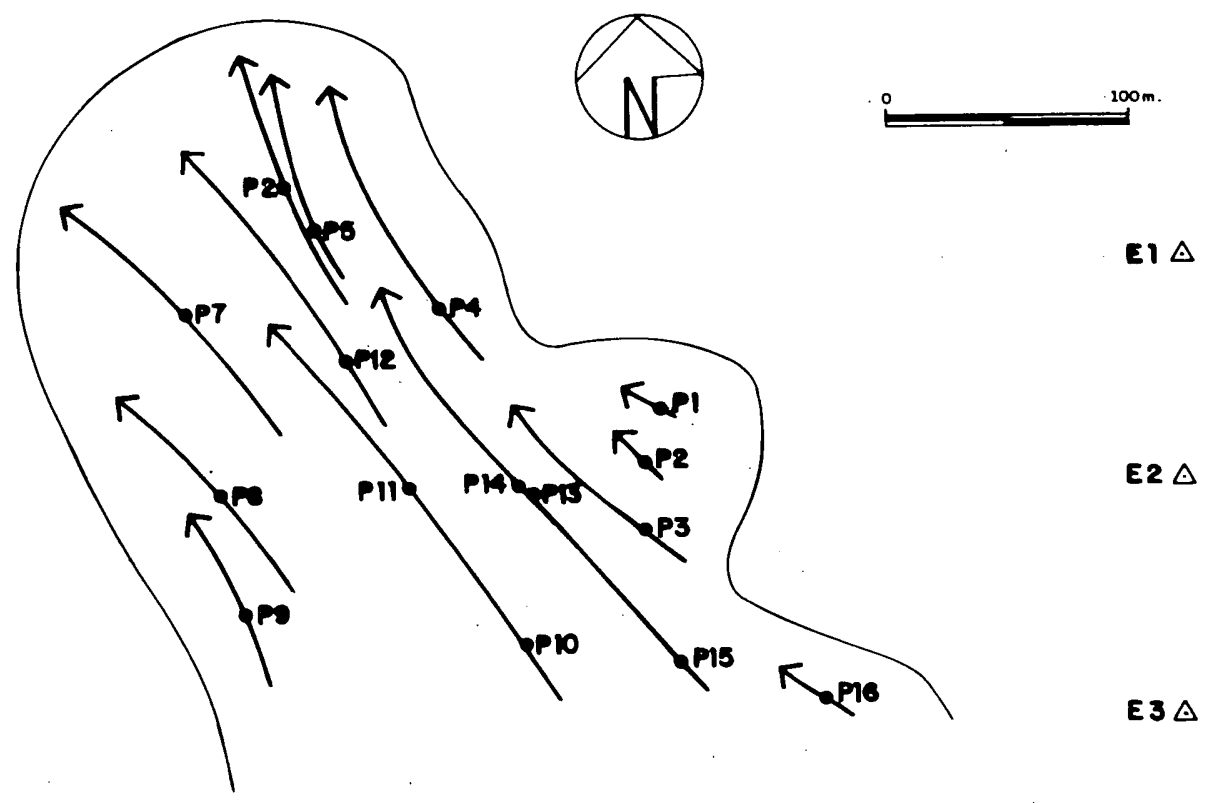

Fig. 2. Direcciones de flujo dominantes en la superficie del glaciar rocoso de las Argualas.

- El conjunto de técnicas aplicadas ha permitido realizar la primera comprobación precisa de movimiento sobre los glaciares rocosos y, por tanto, de la presencia de glaciares rocosos activos en Los Pirineos. Se constata, de este modo, y una vez más, la éxistencia de enérgicos procesos periglaciares en la alta montaña pirenaica por encima de los $2.600 \mathrm{~m}$.

\section{Conclusiones}

De los estudios realizados hasta la actualidad sólo se puede obtener una conclusión definitiva: El glaciar rocoso de las Argualas es un glaciar

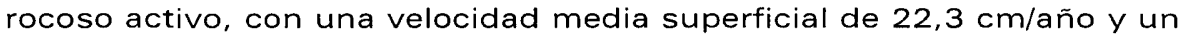
flujo característico de los glaciares rocosos, con un sector central y frontal más activo, y los márgenes más lentos.

El método de trabajo utilizado para el análisis y control superficial del glaciar rocoso de las Argualas, ya aplicado por otros investigadores en aparatos de diferentes lugares del mundo, se muestra válido una vez contrastado por las observaciones continuadas, dados los resultados homogéneos. La continuidad de las observaciones nos permitirá obtener 
resultados útiles para el conocimiento de la dinámica y evolución en detalle del glaciar rocoso de las Argualas.

Agradecimientos. Los Departamentos de "Geografía, Urbanismo y Ordenación de Territorio" e "Ingeniería Geográfica y Expresión Gráfica" de la Universidad de Cantabria y el Programa E.R.H.I.M. del M.O.P.T.M.A. han apoyado y financiado los trabajos cartográficos y de campo para la realización de este trabajo.

\section{Referencias}

Giardino, J.D., Vitek, J.R. (1987): Rock glaciers: a review of the knowledge base. En Giardino Schroder Vitek (eds.), Rock glaciers. Allen and Unwin, Boston: 1-26.

Haeberli, W. (1985): Creep of mountain permafrost: internal structure and flow of alpine rock glaciers. Mitteilungen der Versuchsanstalt für Wasserbau, Hidrologie and glaciologie, Zurich, $142 \mathrm{p}$.

Martínez de Pisón, E. y Arenillas, M.(1988): Los glaciares actuales del Pirineo español. La nieve en el Pirineo español, M.O.P.U. Madrid: 29-98.

Sạn José, J.J.; Agudo, C.; Serrano, E.; Silio, F.(1992): Auscultación topográfica y fotogramétrica del glaciar rocoso de las Argualas (Pirineo aragonés): datos preliminares. Estudios de geomorfología en España, II Reunión Nacional de Geomorfología, S.E.G., Murcia: 423-431.

Serrano, E. y Rubio, V.(1989): El glaciar rocoso activo de las Argualas (Pirineo aragonés). Ería, no 19-20: 195-198. 